\title{
Analysis of the Thomas-Fermi-von Weizsäcker Equation for an Infinite Atom Without Electron Repulsion
}

\author{
Elliott H. Lieb* \\ Departments of Mathematics and Physics, Princeton University, P.O.B. 708, Princeton, NJ 08544, USA
}

\begin{abstract}
The equation

$$
\left\{-\Delta+|\psi(x)|^{2 p-2}-|x|^{-1}\right\} \psi(x)=0
$$

in three dimensions is investigated. Uniqueness and other properties of the positive solution are proved for $3 / 2<p<2$. There are two physical interpretations of this equation for $p=5 / 3$ : (i) As the TFW equation for an infinite atom without electron repulsion; (ii) The positive solution, $\psi$, suitably scaled, is asymptotically equal to the solution of the TFW equation for an atom or molecule with electron repulsion in the regime where the nuclear charges are large and $x$ is close to one of the nuclei.
\end{abstract}

\section{Introduction}

The equation to be analyzed here of primary physical interest is

$$
\left\{-\Delta+|\psi(x)|^{4 / 3}-|x|^{-1}\right\} \psi(x)=0
$$

in three dimensions and with $\psi$ real valued. (1.1) was introduced in [9], wherein it was asserted without proof that (1.1) has a unique, positive solution. The present paper contains that proof. If $z, \gamma, A>0$ and

$$
\tilde{\psi}(z, \gamma, A, x) \equiv\left(z^{2} / A \gamma\right)^{3 / 4} \psi(z x / A),
$$

then

$$
\left\{-A \Delta+\gamma|\tilde{\psi}(x)|^{4 / 3}-z|x|^{-1}\right\} \tilde{\psi}(x)=0 .
$$

and conversely. Thus, (1.3) and (1.1) are equivalent problems.

(1.3) is to be compared with the Thomas-Fermi-von Weizsäcker (TFW) equation for a molecule $[2-4,9,10,13,14]$ :

$$
\left\{-A \Delta+\gamma|\hat{\psi}(x)|^{4 / 3}-V(x)+\left(|x|^{-1} * \hat{\psi}^{2}\right)(x)\right\} \hat{\psi}(x)=-\mu \hat{\psi}(x),
$$

* Work partially supported by U.S. National Science Foundation grant PHY-7825390 A02 
with $V(x)=\sum_{j=1}^{k} z_{j}\left|x-R_{j}\right|^{-1}$ being the potential of $k$ nuclei of charges $z_{j} \geqq 0$ located at $R_{j}$. The term $|x|^{-1} * \hat{\psi}^{2}$ is called "the electron respulsion." (1.3) has a unique positive solution (denoted by $\hat{\psi}$ ) for all $\mu \geqq 0[2,3,9] .-\mu$ is the "chemical potential." For all $\mu \geqq 0, \psi \in L^{2}\left(\mathbb{R}^{3}\right)[3,9]$.

(1.3) is seen to be the TFW equation for an atom $\left(k=1, z_{1}=z, R_{1}=0\right)$, but with the electron repulsion omitted. It will be shown here that (1.1), which is equivalent to (1.3), also has a unique positive solution, $\psi$, and $F(\psi)<\infty$ [cf. (1.8)]. The fact that $\mu=0$ in (1.1) and (1.3) means that the electron number, $\lambda=\int \tilde{\psi}^{2}$, is maximal. We shall see that in this case [for (1.1) and (1.3) but not (1.4)], $\lambda=\infty$, which is physically reasonable since the maximum electron number for the quantum mechanical Bohr atom (without repulsion) is also infinity.

If the foregoing were the only interpretation of (1.3) it would not be especially interesting. However, (1.3) has another interpretation as proved in [9]: Consider the full TFW equation, (1.4), with the scaling $z_{j}=a z_{j}^{0}, R_{j}=a^{-1 / 3} R_{j}^{0}$ and $\lambda=a \lambda^{0}$ with $\lambda^{0}>0$. If $a \rightarrow \infty$ (with $A, \gamma$ fixed), and if $x$ is close to one of the $R_{j}$ then $\hat{\psi}(x) \rightarrow \tilde{\psi}\left(x-R_{j}\right)$ (with $\left.z \equiv z_{j}\right)$ in the following sense: Fix $y \in \mathbb{R}^{3}$ then

$$
\lim _{a \rightarrow \infty} z_{j}^{-3 / 2} \hat{\psi}\left(R_{j}+z_{j}^{-1} y\right)=\lim _{a \rightarrow \infty} z_{j}^{-3 / 2} \tilde{\psi}\left(z_{j}^{-1} y\right)=(A \gamma)^{-3 / 4} \psi(y / A),
$$

where $\psi$ is the positive solution of (1.1) and with the convergence being pointwise and in $L_{\mathrm{loc}}^{1}$. [Note that the second expression in (1.5) is, in fact, independent of $a$. The right side of (1.5) is independent of $\lambda$.]

Not only does $\hat{\psi} \rightarrow \tilde{\psi}$ as in (1.5) but also the difference between the TFW energy and the TF energy is given, to leading order in $a$, by $\psi$ :

$$
E^{\mathrm{TFW}}-E^{\mathrm{TF}}=D \sum_{j=1}^{k} z_{j}^{2}+o\left(a^{2}\right)
$$

where the constant $D$ is given by [9]

$$
D=A^{1 / 2} \gamma^{-3 / 2} F(\psi),
$$

and where the functional $F$ is defined generally for all real $\psi$ by

$$
F(\psi)=\int|\nabla \psi|^{2}+\int k(\psi(x), x) d x
$$

with

$$
k(\psi, x) \equiv \frac{3}{5}|\psi|^{10 / 3}-|x|^{-1} \psi^{2}+\frac{2}{5}|x|^{-5 / 2} \geqq 0 .
$$

Note that $k(\psi, x)>0$ and $k(\psi, x)=0$ if and only if $\psi=|x|^{-3 / 4}$.

The positive solution of (1.1) has been evaluated numerically [8] with the result that

$$
\begin{aligned}
& \psi(0)=0.9701330 \\
& F(\psi)=17.1676 .
\end{aligned}
$$

Generalization. Equation (1.1) has the following obvious generalization

$$
\Delta \psi(x)=\left\{|\psi(x)|^{2 p-2}-|x|^{-1}\right\} \psi(x)
$$


with $p>1$. The physical case, (1.1), corresponds to $p=5 / 3$. The TFW equation, (1.4) can be generalized in the same way; this was in fact done in $[2,3,9]$. In TF theory, $p=4 / 3$ and $3 / 2$ play a special role, while $p=4 / 3,3 / 2$, and $5 / 3$ are special in TFW theory [9]. In the analysis here of (1.10), $p=3 / 2$ and 2 are special (Theorems 3-5 and 7-9). Fortunately, the physical value $5 / 3$ is in the range $3 / 2<p<2$ in which all theorems are applicable.

The appropriate energy functional is $(1.8)$, but with $k(\psi, x)$ replaced by

$$
k_{p}(\psi, x)=\frac{1}{p}|\psi|^{2 p}-|x|^{-1} \psi^{2}+\frac{p-1}{p}|x|^{-p /(p-1)} \geqq 0 .
$$

The corresponding $F$ will be denoted by $F_{p}$. However, as will be seen in Sect. III, $F_{p}$ is useful only when $3 / 2<p<2$. It is for this range of $p$ that the existence of a positive solution will be proved.

One of the more amusing technical exercises is in Sect. IV where an asymptotic expansion for $\psi$ is established. While the expansion is heuristically obvious, its proof is not, primarily because $\Delta$ in (1.10) is essentially a singular perturbation.

\section{Properties of Eq. (1.10)}

Initially, (1.10) will be interpreted as a distributional equation. Although our main interest is in positive solutions, we shall not restrict ourselves to such and will assume only that $\psi$ is a real valued function. It will turn out that the class of functions such that $F_{p}(\psi)<\infty$ is the natural class to consider, when $3 / 2<p<2$, but this will not be assumed initially. The only assumption to be understood in all the theorems is that $p>1$ and

$$
\psi \in M=\left\{\psi \mid \nabla \psi \in L_{\mathrm{loc}}^{2}, \psi \in L_{\mathrm{loc}}^{1}\right\} .
$$

We begin with two "local" theorems.

Theorem 1. Let $B$ be a bounded open ball in $\mathbb{R}^{3}$. Let $\psi$ satisfy (1.10) in $B$ in the sense of distributions with $\psi \in L^{1}(B)$ and $\nabla \psi \in L^{2}(B)$. Then

(i) $\psi$ is continuous. More precisely, $\psi \in C^{0, \alpha}(\bar{B})$ for all $0<\alpha<1$. I.e. there is a constant, $C$, such that $|\psi(x)-\psi(y)| \leqq C|x-y|^{\alpha}$ for all $x, y \in B$.

(ii) If $\psi(x) \geqq 0, \forall x \in B$, then either $\psi(x) \equiv 0$ or else $\psi(x)>0, \forall x \in B$.

(iii) If $0 \notin B$ then $\psi \in C^{1,1 / 2}(\bar{B})$.

(iv) If $0 \notin B$ and $\psi(x) \geqq 0, \forall x \in B$, then $\psi$ is real analytic in $B$.

Proof. (i) $\psi \in W^{1,1}$. By the Sobolev imbedding theorem [1],

$$
\begin{aligned}
\psi \in W^{0,3 / 2}=L^{3 / 2} & \Rightarrow \psi \in W^{1,3 / 2} \Rightarrow \psi \in L^{3} \Rightarrow \psi \in W^{1,2} \Rightarrow \psi \in L^{6} \\
& \Rightarrow f \equiv|x|^{-1} \psi \in L^{2-\varepsilon}, \forall \varepsilon>0 ;
\end{aligned}
$$

in particular $f \in L^{q}$ for some $q>3 / 2$.

If $p<5 / 2$ then $\psi \in L^{6} \Rightarrow|\psi|^{2 p-1} \in L^{3 / 2+\varepsilon}$ for some $\varepsilon>0$. Then $\psi \in W^{2, \varepsilon+3 / 2}$ and thus $\psi \in C^{0, \alpha}$ for some $\alpha>0$. Then the right side of (1.10) is in $L^{3-\varepsilon}$, all $\varepsilon>0$, so $\psi \in W^{2,3-\varepsilon}$. Then $\psi \in C^{0, \alpha}$ for all $\alpha<1$. This proves (i) for $p<5 / 2$. 
If $p \geqq 5 / 2$ a different argument is needed. The fact that (1.10) holds as a distributional equation means that the right side [call it $h(x)$ ] must be a distribution and hence must be in $L_{\text {loc }}^{1}$. Since $|x|^{-1} \psi \in L^{1},|\psi|^{2 p-1} \in L_{\text {loc }}^{1}$. Kato's inequality [7], $\Delta|\psi| \geqq(\operatorname{sgn} \psi) \Delta \psi$ as distributions (with $\operatorname{sgn} \psi=|\psi| / \psi$ if $\psi \neq 0$ and $\operatorname{sgn} \psi=0$ if $\psi=0$ ), then implies that

$$
\Delta|\psi| \geqq(\operatorname{sgn} \psi)\left\{|\psi|^{2 p-2}-|x|^{-1}\right\} \psi=|\psi|^{2 p-1}-|x|^{-1}|\psi| .
$$

Therefore $-\Delta|\psi| \leqq|x|^{-1}|\psi| \equiv f$. A result of Stampacchia [12, Theoréme 5.2] is that if $f \in L_{\mathrm{loc}}^{q}$ with $q>3 / 2$ then $|\psi| \in L_{\mathrm{loc}}^{\infty}$. But our $f$ satisfies this condition. Returning to (1.10), $h \in L_{\text {loc }}^{3-\varepsilon}, \varepsilon>0$, and (i) is thus proved as before.

(ii) We have $\Delta \psi=g \psi$ with $g \in L^{q}$ and $q>3 / 2$. By the Harnack inequality (cf. [5]), $\psi \equiv 0$ or $\psi(x)>0, \forall x$. [1]).

(iii) $\psi, \Delta \psi \in L^{\infty}$ and $\nabla \psi \in L^{2} \Rightarrow \psi \in W^{2,2} \Rightarrow \nabla \psi \in L^{6} \Rightarrow \psi \in W^{2,6} \Rightarrow \psi \in C^{1,1 / 2}$ (see

(iv) Assume $\psi(x)>0$, whence $|\psi|^{2 p-1}$ has as many derivatives as $\psi$ has. By a bootstrap argument $\psi$ is $C^{\infty}$ (see [10, Theorem IV.5]). Since $|x|^{-1}$ is real analytic for $x \neq 0$, by [11, Theorem 5.8.6] $\psi$ is real analytic.

Theorem 2 (cusp condition [6]). Assume the hypotheses of Theorem 1 and also that $0 \in B$. Then

$$
\psi(0)=-2 \lim _{r \downarrow 0} \int \Omega \cdot \nabla \psi(r \Omega) d \Omega,
$$

where $d \Omega$ is the normalized invariant measure on the unit sphere. In particular, if $\psi$ is spherically symmetric about zero, then

$$
\psi(0)=-2 \lim _{r \downarrow 0} d \psi(r) / d r .
$$

Proof. Simply integrate (1.10) by parts.

Theorem 3. Assume that $p \geqq 3 / 2$. Suppose $\psi$ satisfies (1.10) in the sense of distributions on all of $\mathbb{R}^{3}$. Then $|\psi(x)|<|x|^{-1 /(2 p-2)}$.

Remark. Some condition on $p$ is needed and we believe $p \geqq 3 / 2$ is the right one. If $p<3 / 2$ there cannot be any positive $\psi$ satisfying both $(1.10)$ and $\psi(x) \leqq|x|^{-1(2 p-2)}$. For then $0 \leqq h=-\Delta \psi \leqq|x|^{(1-2 p) /(2 p-2)} \equiv f(x)$, where $-h$ is the right side of (1.10). Since $g \equiv|x|^{-1} * f$ is finite for $|x|>0$ and $g(x) \rightarrow 0$ as $|x| \rightarrow \infty, \psi=4 \pi|x|^{-1} * h$. Since $h \neq 0$, this implies that $\psi(x) \geqq c|x|^{-1}$ for $|x|>$ some $R$ and $c>0$. This is a contradiction. Thus, $p \geqq 3 / 2$ is the right condition for positive $\psi$. It is possible that (1.10) has no positive solution even when $p=3 / 2$, for in that case $\psi(x)=|x|^{-1}$ satisfies (1.10) everywhere except at the origin.

Proof. By Theorem 1 we can assume that $\psi$ is continuous. Let $b=-1 /(2 p-2) \geqq-1$ (it is here that $p \geqq 3 / 2$ enters). $\Delta|x|^{b} \leqq 0$. If $g(x) \equiv|\psi(x)|-|x|^{b}$ we have, by (2.2), that $\Delta g>0$ on the set $A=\{x \mid g(x)>0\}$. Since $\psi$ is continuous (Theorem 1) we have that (i) $A$ is open; (ii) $0 \notin A$; (iii) $h(x) \equiv \max [g(x), 0]$ is subharmonic on $\mathbb{R}^{3}$, i.e. $\Delta h \geqq 0$. If we knew that $h(x) \rightarrow 0$ as $|x| \rightarrow \infty$ we could then conclude that $h \equiv 0$ and thus that $|\psi(x)| \leqq|x|^{b}$. But this has to be proved. 
We have, in fact, that on $A$

$$
\Delta h \geqq\left\{|\psi|^{-1 / b}-|x|^{-1}\right\}|\psi| .
$$

Since $|\psi|=h+|x|^{b}$ on $A$, and $(\alpha+\beta)^{t}>\alpha^{t}+\beta^{t}$ for $\alpha, \beta \geqq 0, t \geqq 1$,

$$
\Delta h \geqq h^{2 p-1} \quad \text { on } \quad \mathbb{R}^{3} .
$$

Now let $f(x)=f(|x|)$ be the spherical average of $h$, i.e. $f(r)=\int h(\Omega r) d \Omega$ with $d \Omega$ being the normalized invariant measure on the unit sphere. By averaging (2.6), and using av $\left\{h^{t}\right\} \geqq\{\operatorname{av} h\}^{t}$ for $t \geqq 1$, we have that $\Delta f \geqq f^{2 p^{-1}}$. With $r=|x|$, let $r f(r) \equiv u(r)$, whence

$$
u^{\prime \prime} \geqq r^{-2 p+2} u^{2 p-1} \quad \text { for } \quad r>0
$$

Assume that $u(r) \neq 0$. Since $0 \notin A$, there is a $R_{0}>0$ such that $u(r)=0$ for $0 \leqq r<R_{0}$. Since $u^{\prime \prime} \geqq 0$ and $u \geqq 0, u$ is continuous, convex and non-decreasing in $r$. Therefore for some $R_{1}>0, u\left(R_{1}\right)>0$ and $u^{\prime}\left(R_{1}\right)>0$. Thus $u(r)>b r$ for $r>$ some $R_{2}$ and with $b>0$.

Let $w_{R}(x) \equiv \sigma R(R-r)^{2 b}$ be defined on $D_{R}=\{r \mid 0<r<R\}$ with $\sigma^{2 p-2}$ $=p(p-1)^{-2}$. On $D_{R}, w_{R}$ satisfies $w_{R}^{\prime \prime} \leqq r^{-2 p+2} w_{R}^{2 p-1}$. Since $u(r)>b r$ for large $r$, there is some $R$ such that $w_{R}(R / 2)<u(R / 2)$. Consider $m(r) \equiv u(r)-w_{R}(r)$ on $D_{R}$ and let $E=\{r \mid m(r)>0,0<r<R\}$. Clearly, (i) $m$ is continuous and $m^{\prime \prime}>0$ on $E$; (ii) $E$ is nonempty and open; (iii) $\bar{E} \subset D_{R}$ [since $u(r)=0$ for $r<R_{0}$ and $w_{R}(r) \rightarrow \infty$ as $r \rightarrow R$ ]. Therefore $m$ has its maximum on $\partial E$, but $m=0$ on $\partial E$. Therefore $E$ is empty and we have a contradiction.

Thus $u \equiv 0$, which implies that $f \equiv 0 \Rightarrow h \equiv 0 \Rightarrow \psi(x) \leqq|x|^{b}$. We have to prove that $g(x)<0$ everywhere. The Harnack inequality argument used in Theorem 1 implies that either $g \equiv 0$ or $g(x)<0$, all $x$. Since $g(x)<0$ for $x$ near zero, the result is proved.

The following complements Theorem 3.

Theorem 4. Suppose $\psi(x)>0$ satisfies $(1.10)$ on $\mathbb{R}^{3}$ and $\psi$ is spherically symmetric. If $p<2$ then $\psi(x) \geqq(A+|x|)^{-1(2 p-2)}$ with $A=1 /(p-1)$.

Remark. The hypothesis that $\psi$ is spherically symmetric is not really necessary but it simplifies the proof. It is assumed here because Theorem 5 states that any positive solution is necessarily spherically symmetric when $3 / 2 \leqq p<2$.

Proof. Let $f(r)=(A+r)^{b} \quad$ with $\quad b=-1 /(2 p-2)$. Then $\Delta f=W f \quad$ with $W(r)=b(b-1)(A+r)^{-2}+(2 b / r)(A+r)^{-1} . \quad W(r)>f(r)^{2 p-2}-r^{-1} . \quad$ Let $B=\{r>0 \mid \psi(r)<f(r)\}$, which is open and which is assumed to be non-empty. Let $D$ be a connected component of $B$. Thus $D=(s, t)$ with $0 \leqq s<t$. Let $h=f-\psi>0$ on $D$. On $D, W(r)>\psi(r)^{2 p-2}-r^{-1}$, so

$$
(\Delta-W) h(r)>0, \quad \text { all } \quad r \in D \text {. }
$$

First, assume $t$ is finite. Multiply (2.8) by $f$ and integrate over the shell $s<|x|<t$. An integration by parts gives

$$
K(t)-K(s)>0
$$


with $K(r)=r^{2} f(r)^{2}(h / f)^{\prime}(r) .(h / f)^{\prime}(t) \leqq 0$ since $h(t)=0$ and $h(r)>0$ for $s<r<t$. Thus $K(t) \leqq 0$. Likewise, if $s>0 K(s) \geqq 0$ and therefore (2.8) is a contradiction. If $s=0$ then $K(s)=0$ since $\psi^{\prime}$ and $f^{\prime}$ are bounded near $r=0$.

Now assume $t=\infty$. Integrate (2.9) over $s<|x|<T$. (2.8) holds as before (with $T$ ) and $K(s) \geqq 0$. Since $p<2, r f(r)^{2} \rightarrow 0$ as $r \rightarrow \infty$. $0<(h / f)(r)<1$ on $D$, so there is a sequence $\left\{T_{n}\right\}$, with $T_{n} \rightarrow \infty$, such that $T_{n}(h / f)^{\prime}\left(T_{n}\right) \rightarrow 0$. Thus $K\left(T_{n}\right) \rightarrow 0$ and (2.8) is again a contradiction.

Theorem 5. There is at most one $\psi \neq 0$ that satisfies $(1.10)$ on $\mathbb{R}^{3}$ in the sense of distributions with the properties that $\psi(x) \geqq 0$, all $x$ and $\psi \in L^{6}\left(\mathbb{R}^{3}\right)$. (By Theorem 3 , the second property is automatic if $3 / 2 \leqq p<2$.)

Remark. It is not at all clear whether or not $p \geqq 2$ is special. If it is additionally assumed that $\nabla \psi \in L^{2}\left(\mathbb{R}^{3}\right)$ then by Theorem 3 and a Sobolev inequality, $\psi \in L^{6}$ when $p \geqq 3 / 2$. In Sect. III it will be shown that there is a positive solution to (1.10) for $3 / 2<p<2$, and this solution indeed has $\nabla \psi \in L^{2}\left(\mathbb{R}^{3}\right)$. However, the methods of Sect. III are not applicable for $p \geqq 2$.

Proof. Let $\psi$ satisfy (1.10) with the stated properties. By Theorem 1, $\psi$ is continuous and $\psi(x)>0$, all $x$. If $g \in C_{0}^{\infty}$, then $-\int \frac{\Delta \psi}{\psi}|g|^{2} \leqq \int|\nabla g|^{2}$. (This fact, which was used in [3], follows by integrating by parts and using the Cauchy-Schwarz inequality.) Thus, for any real $g \in C_{0}^{\infty}$,

$$
L_{\psi}(g)=\int|\nabla g|^{2}+\int W_{\psi}(x)|g(x)|^{2} d x \geqq 0,
$$

where $W_{\psi}(x)=-|x|^{-1}+\psi(x)^{2 p-2}$. Let $f_{n}$ be a sequence of spherically symmetric $C_{0}^{\infty}$ functions with the properties : (i) $0 \leqq f_{n}(x) \leqq 1$; (ii) $f_{n}(x)=1$ for $2 / n<|x|<n$; (iii) $f_{n}(x)=0$ for $0 \leqq|x| \leqq 1 / n$ and $|x| \geqq 2 n$; (iv) $\int\left|\nabla f_{n}\right|^{3} \leqq A$ for some fixed $A$. Such a sequence is easy to construct. Let $g_{n}(x)=f_{n}(x) \psi(x) . g_{n} \in C_{0}^{\infty}$ by Theorem 1. $\int\left|\nabla g_{n}\right|^{2}$ $=-\int f_{n}^{2} \psi \Delta \psi+\int \psi^{2}\left|\nabla f_{n}\right|^{2}$. Thus, $L_{\psi}\left(g_{n}\right)=\int \psi^{2}\left|\nabla f_{n}\right|^{2} \equiv T_{n}$. Let $\chi_{n}(x)=0$ if $2 / n$ $\leqq|x| \leqq n$ and $\chi_{n}(x)=1$ otherwise. Then $T_{n}=\int \chi_{n} \psi^{2}\left|\nabla f_{n}\right|^{2} \leqq\left\|\chi_{n} \psi\right\|_{6}^{2}\left\|\nabla f_{n}\right\|_{3}^{2}$. Since $\chi_{n} \rightarrow 0$ pointwise and $\psi \in L^{6}, T_{n} \rightarrow 0$ as $n \rightarrow \infty$.

Now let $\psi_{1}$ and $\psi_{2}$ be two different solutions to (1.10) with the stated properties. Denote the corresponding two functionals in (2.10) by $L_{1}$ and $L_{2}$ and the corresponding sequences by $g_{n}^{1}=f_{n} \psi_{1}$ and $g_{n}^{2}=f_{n} \psi_{2}$. Then $0 \leqq L_{1}\left(g_{n}^{2}\right)+L_{2}\left(g_{n}^{1}\right)$ $=L_{1}\left(g_{n}^{1}\right)+L_{2}\left(g_{n}^{2}\right)-\int\left(\psi_{1}^{2 p-2}-\psi_{2}^{2 p-2}\right)\left(\psi_{1}^{2}-\psi_{2}^{2}\right) f_{n}^{2}$. Since $L_{1}\left(g_{n}^{1}\right)$ and $L_{2}\left(g_{n}^{2}\right) \rightarrow 0$, the right side of this inequality is negative for large $n$ if $\psi_{1} \neq \psi_{2}$.

Remark. If $\psi \geqq 0$ is unique then $\psi$ is obviously spherically symmetric. Therefore the following theorem is applicable if $3 / 2 \leqq p<2$.

Theorem 6. If $\psi \geqq 0$ satisfies (1.10), the bound of Theorem 3, and if $\psi$ is spherically symmetric, then $\psi(r)$ is a strictly decreasing function of $r$.

Proof. $\Delta \psi \leqq 0$ so $\psi$ is superharmonic. Therefore the minimum of $\psi(x)$ in the set $|x| \leqq R$ must occur at $R$. If $\psi(r)=\psi(R), r<R$, then $\psi(x)$ is a constant for $r \leqq|x| \leqq R$, but this does not satisfy (1.10). 


\section{Existence of a Positive Solution of Eq. (1.10) for $3 / 2<p<2$}

In Sect. II it was shown that any positive distributional solution to (1.10) with $\psi \in M$ has certain nice properties, especially when $p \geqq 3 / 2$. If $3 / 2 \leqq p<2$ the solution is unique. In this section it will always be assumed that $3 / 2<p<2$ (note the inequality $p>3 / 2$ ). A positive solution will be shown to exist on $\mathbb{R}^{3}$ under this condition.

An interesting open question is whether (1.10) has solutions, positive or otherwise, when $p \leqq 3 / 2$ or $p \geqq 2$. The method given here sheds no light on this question.

Consider the functional $F_{p}$ given by (1.8), (1.11) and the class of measurable functions

$$
G_{p}=\left\{\psi \mid \nabla \psi \in L^{2}, F_{p}(\psi)<\infty\right\} .
$$

$G_{p}$ is not empty because

$$
f_{p}(x)=\left(1+|x|^{2}\right)^{-1 / 4(p-1)}
$$

is in $G_{p}$ for $3 / 2<p<2$. We also define

$$
\begin{gathered}
W_{p}(\psi)=\int k_{p}(\psi(x), x) d x \geqq 0, \\
E_{p}=\inf \left\{F_{p}(\psi) \mid \psi \in G_{p}\right\} .
\end{gathered}
$$

Remark. The condition $3 / 2<p<2$ results from the requirement that $G_{p}$ be nonempty. Note that $k_{p}(\psi, x)$ has the form $|x|^{-p /(p-1)} h_{p}\left(|\psi||x|^{1 /(2 p-2)}\right)$ and that $h_{p}(a)=0$ if and only if $a=1$. Suppose $p \geqq 2$. If $k_{p}(\psi(x), x)$ is to be integrable at infinity then $\psi(x) \sim|x|^{-1 /(2 p-2)}$. But then $|\nabla \psi|^{2}$ is not integrable at infinity. If $p \leqq 3 / 2$ then, for a similar reason, $\psi(x) \sim|x|^{-1 /(2 p-2)}$ near $x=0$, but then $|\nabla \psi|^{2}$ is not integrable.

Theorem 7. Let $3 / 2<p<2$. There exists $\psi \in G_{p}$ such that (i) $F_{p}(\psi)=E_{p}$; $\psi(x) \geqq 0$; (iii) $\psi$ satisfies (1.10) in the sense of distributions.

Proof. Let $\psi_{n}$ be a minimizing sequence for $F_{p}(\psi)$. $W_{p}(|\psi|)=W_{p}(\psi)$ and $\int|\nabla \psi|^{2}$ $\geqq \int(\nabla|\psi|)^{2}$, so we can assume $\psi_{n}(x) \geqq 0$, all $x$. Let $b=-(2 p-2)^{-1}$. For $\psi \geqq 0$, $k_{p}(\psi, x) \geqq C_{p}|x|^{-1}\left[\psi-|x|^{b}\right]^{2}$ with $C_{p}>0$. Therefore, if $\psi \in G_{p}$ then $|x|^{-1 / 2} \psi$ $-|x|^{b-1 / 2} \in L^{2}$. But $|x|^{b-1 / 2} \in L_{\mathrm{loc}}^{2}$ so $|x|^{-1 / 2} \psi \in L_{\mathrm{loc}}^{2} \Rightarrow \psi \in L_{\mathrm{loc}}^{2} \Rightarrow \psi \in W^{1,2}(B)$ for any bounded ball, $B$. Since $F_{p}\left(\psi_{n}\right) \rightarrow E_{p}, \nabla \psi_{n}$ is bounded in $L^{2}\left(\mathbb{R}^{3}\right)$ and $\psi_{n}$ is bounded in $W^{1,2}(B)$. By the Rellich-Kondrachov theorem [1], a bounded set in $W^{1,2}(B)$ is compactly imbedded in $L^{2}(B)$. By passing to a subsequence we can assume $\psi_{n}$ has a limit, $\psi$, in $L^{2}(B)$. By taking a further subsequence, we can assume $\psi_{n} \rightarrow \psi$ pointwise. This can be done for every $B$, so we can assume $\psi_{n} \rightarrow \psi$ pointwise in $\mathbb{R}^{3}$. By passing to a further subsequence we can assume, using the Banach-Alaoglu theorem, that $\nabla \psi_{n} \rightarrow f$ weakly in $L^{2}\left(\mathbb{R}^{3}\right)$. Clearly, $f=\psi$. Therefore $\lim \inf \int\left|\nabla \psi_{n}\right|^{2}$ $\geqq \int|\nabla \psi|^{2}$ and, by Fatou's lemma, liminf $\int k_{p}\left(\psi_{n}(x), x\right) \geqq \int k_{p}(\psi(x), x)$. Thus $F_{p}(\psi) \leqq E_{p}$, so $\psi$ minimizes.

Now $k_{p}(\psi(x), x) \in L^{1}$. By the above $|x|^{-1} \psi^{2} \in L_{\mathrm{loc}}^{1} \Rightarrow|x|^{-1} \psi \in L_{\mathrm{loc}}^{1}$. Since $k_{p}(\psi(x), x) \in L^{1}, \psi^{2 p} \in L_{\mathrm{loc}}^{1}$, and thus $\psi^{2 p-1} \in L_{\mathrm{loc}}^{1}$. Hence, the right side of (1.10) is a distribution. 
Let $\eta \in C_{0}^{\infty}$ and replace $\psi$ by $\psi_{t}=\psi+t \eta$. Let $E(t)=E_{p}\left(\psi_{t}\right)$, whence $d E / d t=0$ at $t=0 . \int\left|\nabla \psi_{t}\right|^{2}$ is differentiable with derivative $2 \int \nabla \psi \cdot \nabla \eta$ at $t=0$. By dominated convergence, $W_{p}\left(\psi_{t}\right)$ can be differentiated under the integral sign, whence $0=\int \nabla \eta \cdot \nabla \psi+\int \eta\left\{|\psi|^{2 p-2}-|x|^{-1}\right\} \psi$. This is Eq. (1.10) in the sense of distributions.

The $\psi$ given by Theorem 7 is unique (Theorem 5). $\psi$ satisfies two sum rules, one of which arises from the fact that $\psi$ minimizes $F_{p}$. Let us define the following integrals :

$$
\begin{aligned}
& I_{1}=\int|\nabla \psi(x)|^{2} d x, \\
& I_{2}=\int\left\{|x|^{-p /(p-1)}-\psi(x)^{2 p}\right\} d x, \\
& I_{3}=\int|x|^{-1}\left\{|x|^{-1 /(p-1)}-\psi(x)^{2}\right\} d x .
\end{aligned}
$$

$I_{1}$ is finite since $\psi \in G_{p} . I_{2}$ and $I_{3}$ are finite (and positive) since $|x|^{-1 /(2 p-2)}>\psi(x)$ $>(A+|x|)^{-1 /(2 p-2)}$, Theorems 3 and 4 , and since $|x|^{-p /(p-1)} \in L_{\mathrm{loc}}^{1}$. Clearly,

$$
F_{p}(\psi)=I_{1}-p^{-1} I_{2}+I_{3}
$$

The physical interpretation of these integrals is the following: $I_{1}$ is the gradient contribution to the kinetic energy. $I_{2} / p$ is the decrease in the "fermionic" part of the kinetic energy relative to the TF value. $I_{3}$ is the increase in the electron-nucleus potential energy relative to the TF value.

Theorem 8. Let $\psi$ be the minimizing $\psi$ of Theorem 7. Then

$$
I_{1}: I_{2}: I_{3}=2(2-p)(p-1): p(3-p): p^{2}-3 p+4 \text {. }
$$

In particular, for $p=5 / 3$,

$$
I_{1}: I_{2}: I_{3}=1: 5: 4 \text {. }
$$

Proof. If (1.10) is multiplied by $\psi$ and integrated, we find $I_{2}=I_{1}+I_{3}$. Next, consider $\psi_{t}(x) \equiv t^{1 /(2 p-1)} \psi(t x)$ for $t>0$. Since $g(t) \equiv F_{p}\left(\psi_{t}\right)$ has its minimum at $t=1$, $d g(t) / d t=0$ at $t=1$. But $I_{1}\left(\psi_{t}\right)=t^{-1} I_{1}, I_{2}\left(\psi_{t}\right)=t^{(4-2 p) /(p-1)} I_{2}$ and $I_{3}\left(\psi_{t}\right)$ $=t^{(4-2 p) /(p-1)} I_{3}$. The rest is algebra.

Remark. When $p=5 / 3$, Theorem 8 implies the virial theorem. The change in kinetic energy is $\delta T=I_{1}-p^{-1} I_{2}$. The change in potential energy is $\delta V=I_{3}$. Therefore $-2 \delta T=\delta V$, as usual.

\section{Asymptotic Expansion for Large $r(3 / 2<p<2)$}

We shall be concerned here with the unique, positive solution to (1.10) for $3 / 2<p<2$. By Theorems 3 and 4, $\psi(r)=r^{b}+O\left(r^{b-1}\right)$ with $r=|x|$ and $b=-1 /(2 p-2)$. This suggests an asymptotic expansion of the form

$$
\psi(r)=r^{b}\left\{1+\sum_{j=1}^{\infty} a_{j} r^{-j}\right\} .
$$


The coefficients $a_{j}$ are determined as follows. If (4.1) is inserted into the right side of (1.10) and then expanded, the coefficient of $r^{b-1}$ is zero and the coefficient of $r^{b-j-1}(j \geqq 1)$ is of the form

$$
P_{j}\left(A_{j-1}\right)+(2 p-2) a_{j},
$$

where $A_{j} \equiv\left(a_{1}, \ldots, a_{j}\right)$ and $P_{j}$ is a polynomial (with $P_{1} \equiv 0$ ). The coefficient of $r^{b-j-1}$ on the left side is zero for $j=0$ and is

$$
(b-j+2)(b-j+1) a_{j-1}
$$

for $j \geqq 1$, with $a_{0} \equiv 1$. Equating (4.2) and (4.3),

$$
a_{j}=(2 p-2)^{-1}\left[(b-j+2)(b-j+1) a_{j-1}-P_{j}\left(A_{j-1}\right)\right] .
$$

Thus, $a_{j}$ is determined recursively by $a_{1}, \ldots, a_{j-1}$. The first three terms of $\psi$ are thus

$$
\psi(r)=r^{b}\left\{1-r^{-1}(2 p-3)(2 p-2)^{-3}-\frac{1}{2} r^{-2}(2 p-3)(2 p-1)^{2}(2 p-2)^{-6}+O\left(r^{-3}\right)\right\} .
$$

The correctness of (4.1), with the rule (4.4), will be proved here. The chief difficulty is that $\Delta$ is a singular perturbation: The term $a_{j} r^{b-j}$ in $\psi$ generates a term $r^{b-j-1}$ on the right side of (1.10), but it generates $r^{b-j-2}$ on the left. While $\Delta \psi$ thus appears to be relatively small, it is not really small because its coefficients (4.3) grow as $j^{2}$. For this reason the series (4.1) is probably not convergent. In the proof of Theorem $9, \Delta \psi$ is controlled by combining it with the leading term in (4.2), namely $(2 p-2) r^{-1} \psi$.

Theorem 9. The asymptotic expansion (4.1) and (4.4) is correct, i.e. for any J,

$$
\psi(r)=r^{b}\left\{1+\sum_{j=1}^{J} a_{j} r^{-j}\right\}+o\left(r^{b-J}\right)
$$

as $r \rightarrow \infty$.

Proof. The proof is by induction. Theorems 3 and 4 assert that (4.6) is true for $J=0$. Assuming (4.6) holds for some $J \geqq 0$, we will prove that (4.6) holds for $J+1$. Write $\psi=\phi+g$ with $\phi(r)=r^{b}\left\{1+\sum_{j=1}^{J} a_{j} r^{-j}\right\}$. For any $\varepsilon>0$ there is an $R_{\varepsilon}$ such that for all $r>R_{\varepsilon}$ : (i) $\phi(r)>0$, (ii) $g(r) / \phi(r)<\varepsilon$, (iii) $[\phi(r)+g(r)]^{2 p-1}-\phi(r)^{2 p-1} \equiv U(r) g(r)$ with $\left|U(r)-(2 p-1) r^{-1}\right|<\varepsilon r^{-1}$.

Equation (1.10) reads

$$
[\Delta+W(r)] g(r)=h(r),
$$

with $h=\phi^{2 p-1}-r^{-1} \phi-\Delta \phi$ and $W(r)=r^{-1}-U(r)$. Let us examine (4.7) for $r>R_{\varepsilon}$. As $r \rightarrow \infty, h(r)=K r^{b-J-2}+o\left(r^{b-J-2}\right)$ since $a_{1}, \ldots, a_{J}$ satisfy (4.4). Moreover, $K=-(2 p-2) \cdot[$ right side of (4.4) with $j=J+1]$. We also know that $g(r)=o\left(r^{b-J}\right)$ by the induction hypothesis. Finally, $\left|W(r)+(2 p-2) r^{-1}\right|<\varepsilon r^{-1}$ by (iii) above. The point of writing (1.10) as (4.7) is that we now regard $W$ as a fixed function that is close to $-(2 p-2) r^{-1}$. It is true that $W$ "depends on $g$ ", but that information is suppressed. 
Lemma 10 will imply that $g(r)=-K(2 p-2)^{-1} r^{b-J-1}+o\left(r^{b-J-1}\right)$, which is the desired result. To see this, let $z>0$ and define $v_{z}(r)=\exp \left[-2(z r)^{1 / 2}\right]$. Let $W_{z}(r)=-z / r$. Then $\left(\Delta+W_{z}\right) v_{z}=-\frac{3}{2} z^{1 / 2} r^{-3 / 2} v_{z}$. Without loss, assume $K \geqq 0$ [otherwise replace $g$ by $-g$ in (4.7)]. Let $z_{0}=2 p-2$.

Lower Bound for $g$. Pick $0<\varepsilon<z_{0}$ and let $z=z_{0}-\varepsilon$. For $r>R_{\varepsilon}, W(r) \leqq-z / r$ $=W_{z}(r)$. Pick $\delta>0$ and $A \geqq 0$ and let $f(r)=-A v_{z}(r)-(K+\delta) z^{-1} r^{b-J-1}<0$. Then

$$
\begin{aligned}
\left(\Delta+W_{z}\right) f(r)= & \frac{3}{2} A z^{1 / 2} r^{-3 / 2} v_{z}(r)+(K+\delta) r^{b-J-2} \\
& -(K+\delta) z^{-1}(b-J)(b-J-1) r^{b-J-3} .
\end{aligned}
$$

For any fixed $\varepsilon, \delta>0$ we can choose $A \geqq 0$ such that (i) $\left(\Delta+W_{z}\right) f \geqq h=(\Delta+W) g$ for $r>R_{\varepsilon}$, (ii) $g\left(R_{\varepsilon}\right) \geqq f\left(R_{\varepsilon}\right)$. By Lemma 10 [with $B=\left\{r \mid r>R_{\varepsilon}\right\}, g_{1}=g, g_{2}=f, W_{1}=W$, $W_{2}=W_{z}$, and the facts that $g(r)$ and $f(r) \rightarrow 0$ as $r \rightarrow \infty$ and $\left.f(r)<0\right], g(r) \geqq f(r)$ for $r>R_{\varepsilon}$. This implies $\liminf _{r \rightarrow \infty} r^{-b+J+1} g(r) \geqq-(K+\delta) / z$. Since $\delta$ and $\varepsilon$ are arbitrary, $\liminf _{r \rightarrow \infty} r^{-b+J+1} g(r) \geqq-r \rightarrow \infty / z_{0}$.

Upper Bound for $g$. This is similar to the preceding. For $r>R_{\varepsilon},-z / r \geqq W(r)$ $\geqq-q / r$ with $q=z_{0}+\varepsilon$ and $z=z_{0}-\varepsilon$. Let $G(r)=g(r)-A v_{z}(r)-\delta r^{b-J-1}$. Then

$$
\begin{aligned}
(\Delta+W) G(r)= & h(r)+\frac{3}{2} A z^{1 / 2} r^{-3 / 2} v_{z}(r)-A\left(W(r)+z r^{-1}\right) v_{z}(r) \\
& -\delta(b-J)(b-J-1) r^{b-J-3}-\delta W(r) r^{b-J-1}
\end{aligned}
$$

Let $F(r)=-K q^{-1} r^{b-J-1} \leqq 0$, whence

$$
\left(\Delta+W_{q}\right) F(r)<K r^{b-J-2} .
$$

For any fixed $\varepsilon, \delta>0$ we can choose $A \geqq 0$ such that (i) $(\Delta+W) G \geqq\left(\Delta+W_{q}\right) F$ for $r>R_{\varepsilon}$, (ii) $F\left(R_{\varepsilon}\right) \geqq G\left(R_{\varepsilon}\right)$. By Lemma $10 \quad\left(g_{1}=F, g_{2}=G, W_{1}=W_{q}, W_{2}=W\right)$, $F(r) \geqq G(r)$. As before this implies $\limsup _{r \rightarrow \infty} r^{-b+J+1} g(r) \leqq-K / z_{0}$.

These two bounds yield the desired result.

Lemma 10. Let $B \subset \mathbb{R}^{3}$ be an open set, let $g_{i}, i=1,2$, be two functions on $\mathbb{R}^{3}$ which are continuous on some neighborhood of $\bar{B}$, the closure of $B$, and let $W_{i}$ be two functions in $L_{\mathrm{loc}}^{1}(B)$. Suppose that $\left[\Delta+W_{1}\right] g_{1} \leqq\left[\Delta+W_{2}\right] g_{2}$ as distributions on $B$. In addition suppose that (i) $g_{1} \geqq g_{2}$ on the boundary of $B$, (ii) if $B$ is unbounded, then for every $\varepsilon>0$ there exists an $R_{\varepsilon}$ such that $g_{1}(x)-g_{2}(x) \geqq-\varepsilon$ on $\left\{x|x \in B| x \mid, \geqq R_{\varepsilon}\right\}$, (iii) $0 \geqq W_{2}(x) \geqq W_{1}(x)$, all $x \in B$, (iv) $g_{1}(x) \leqq 0$ whenever $g_{1}(x)<g_{2}(x)$. Then $g_{1}(x) \geqq g_{2}(x)$ for all $x \in B$.

Proof. Let $\phi=g_{1}-g_{2}$. Let $D=\{x \mid x \in B, \phi(x)<0\}$, which is open. Then $\Delta \phi \leqq W_{2} g_{2}$ $-W_{1} g_{1}$, and thus $\Delta \phi \leqq 0$ on $D$ by (iii) and (iv). Let $U_{\varepsilon}=\left\{x|| x \mid<R_{\varepsilon}\right\}$. $\phi$ is superharmonic on $D \cap U_{\varepsilon}$, but $\phi=0$ on $\partial D$ and $\phi \geqq-\varepsilon$ on $\partial U_{\varepsilon}$. Thus $\phi \geqq-\varepsilon$ on $D \cap U_{\varepsilon}$. Since $\varepsilon$ is arbitrary and $R_{\varepsilon}$ can be chosen to tend to infinity as $\varepsilon \rightarrow 0, \phi \geqq 0$ on $D$, so $D$ is empty. 


\section{References}

1. Adams, R.A.: Sobolev spaces. New York: Academic Press 1975

2. Benguria, R.: The von Weizsäcker and exchange corrections in Thomas-Fermi theory. Ph. D. thesis, Princeton University 1979 (unpublished)

3. Benguria, R., Brezis, H., Lieb, E.H.: The Thomas-Fermi-von Weizsäcker theory of atoms and molecules. Commun. Math. Phys. 79, 167-180 (1981)

4. Fermi, E.: Un metodo statistico per la determinazione di alcune priorieta dell'atome. Rend. Accad. Naz. Lincei 6, 602-607 (1927)

5. Gilbarg, D., Trudinger, N.: Elliptic partial differential equations of second order. Berlin, Heidelberg, New York: Springer 1977

6. Kato, T.: On the eigenfunctions of many particle systems in quantum mechanics. Commun. Pure Appl. Math. 10, 151-171 (1957)

7. Kato, T.: Schrödinger operators with singular potentials. Isr. J. Math. 13, 135-148 (1973)

8. Liberman, D., Lieb, E.H.: Numerical calculation of the Thomas-Fermi-von Weizsäcker function for an infinite atom without electron repulsion, Los Alamos National Laboratory report (in preparation)

9. Lieb, E.H.: Thomas-Fermi and related theories of atoms and molecules. Rev. Mod. Phys. 53, 603-641 (1981)

10. Lieb, E.H., Simon, B.: The Thomas-Fermi theory of atoms, molecules, and solids. Adv. Math. 23, 22-116 (1977)

11. Morrey, C.B., Jr.: Multiple integrals in the calculus of variations. Berlin, Heidelberg, New York: Springer 1966

12. Stampacchia, G.: Equations elliptiques du second ordre à coefficients discontinus. Montréal: Presses de l'Univ. 1965

13. Thomas, L.H.: The calculation of atomic fields. Proc. Camb. Phil. Soc. 23, 542-548 (1927)

14. von Weizsäcker, C.F. : Zur Theorie der Kernmassen. Z. Phys. 96, 431-458 (1935)

Communicated by R. Jost

Received November 11, 1981 
